\title{
MULTIPLIERS OF HANKEL MATRICES
}

\author{
S. J. POREDA, A. K. KABLY and J. W. WARD
}

\section{Introduction}

Let $\left\{\gamma_{j}\right\}_{j=1}^{\infty}$ be any sequence of complex numbers. The infinite matrix $\Gamma$ given by

$$
\Gamma=\left(\begin{array}{cccc}
\gamma_{1} & \gamma_{2} & \gamma_{3} & \ldots \\
\gamma_{2} & \gamma_{3} & \gamma_{4} & \ldots \\
\gamma_{3} & \gamma_{4} & \gamma_{5} & \ldots \\
\vdots & \vdots & \vdots & \vdots
\end{array}\right)=\left(\gamma_{j+k-1}\right)
$$

is called a (infinite) Hankel matrix. If, when we let $\Gamma$ operate on $l^{2}$ in the usual way, we obtain a bounded linear operator from $l^{2}$ into $l^{2}$; we say $\Gamma \in G^{\infty}$. The following theorem completely characterizes $G^{\infty}$.

Theorem 1.1 (Nehari) [10]. Given $\Gamma=\left(\gamma_{j+k-1}\right)$ then $\Gamma \in G^{\infty}$ if and only if there exists $F \in L^{\infty}$ such that

for $k=1,2, \ldots$ Moreover,

$$
\gamma_{k}=\frac{1}{2 \pi} \int_{2 \pi}^{0} e^{i k \theta} F(\theta) d \theta
$$

$$
\|\Gamma\| \equiv \sup _{\substack{\xi \in l^{2} \\\|\xi\|_{2}>0}} \frac{\|\Gamma \xi\|_{2}}{\|\xi\|_{2}}=\operatorname{dist}\left(F, H^{\infty}\right) \equiv \inf _{h \in H^{\infty}}\|F-h\|_{\infty} .
$$

Using the notation of the above theorem it will be convenient to identify an arbitrary matrix in $G^{\infty}$ with $\Gamma_{F}, F \in L^{\infty}$. Of course $F$ is not unique but for our purposes, any member of the corresponding coset in $L^{\infty} / H^{\infty}$ will suffice.

Nehari's theorem establishes a connection between the problem of approximating a given $L^{\infty}$ function by $H^{\infty}$ functions and, that of determining the norm of a corresponding operator on $l^{2}$. A generalization of the first problem that has been investigated by several authors is to consider approximating an $L^{\infty}$ function $F$ by functions of the form $h(z) / p_{n}(z)$ where $h \in H^{\infty}$ and $p_{n}$ is a polynomial of degree $n$. It is this more general approximation problem that draws attention to studying multipliers of Hankel matrices for as is shown in Section 4, it is equivalent to finding a multiplier of a certain type whose product with $\Gamma_{F}$ has minimal norm. The problem of characterizing all multipliers thus arises and is solved in Section 2 where we show that these multipliers are isometrically isomorphic to $H^{\infty}$. In Sec- 
tion 3 we relate the properties of a given multiplier as an operator on $G^{\infty}$ to the properties of the corresponding $H^{\infty}$ function. We conclude in Section 5 by making some remarks and discussing an open problem.

One may wish to compare this characterization of the multipliers of Hankel matrices with the corresponding theorem for Toeplitz matrices which was given by Brown and Halmos [5]. Although it is not explicitly stated in their paper, the multipliers in both cases are identical, that is, as in the case of Hankel matrices, the space of multipliers of Toeplitz matrices is isometrically isomorphic to $H^{\infty}$. The similarity between these two spaces of matrices seems to stop there with regard to their multipliers for there doesn't seem to be any theorem for Toeplitz matrices which corresponds to Nehari's theorem. It is also somewhat interesting to compare the proofs of these two characterizations for in the case of Toeplitz matrices it is somewhat elementary whereas in the case of Hankel matrices, it is definitely nontrivial.

\section{Multipliers}

An infinite matrix $M=\left(\alpha_{i j}\right)_{i, j}$ is said to be a multiplier of $G^{\infty}$, the set of all (bounded) infinite Hankel matrices, if for every $\Gamma \in G^{\infty}, M \Gamma \in G^{\infty}$ where $M \Gamma$ is the usual matrix product of $M$ and $\Gamma$.

If $M$ is such a multiplier we define the norm of $M$ by

$$
\|M\|=\sup _{\substack{\Gamma \in G^{\infty} \\\|\Gamma\| \leqq 1}}\|M \Gamma\| .
$$

If $\|M\|<\infty$ we call $M$ a bounded multiplier of $G^{\infty}$. Let $\mathscr{M}$ denote the set of all such multipliers. We shall see in Section 5, that every multiplier is apriori bounded.

It is not difficult to show that a necessary condition for $M$ to be a multiplier of $G^{\infty}$ is that $M$ be of the form: $M=\left(a_{j-i}\right)_{i, j}$ where $\alpha_{-k}=0$ for $k=1,2, \ldots$,

(2.1) i.e.

$$
M=\left(\begin{array}{cccc}
\alpha_{0} & \alpha_{1} & \alpha_{2} & \ldots \\
0 & \alpha_{0} & \alpha_{1} & \ldots \\
0 & 0 & \alpha_{0} & \ldots \\
\vdots & \vdots & \vdots & \ddots
\end{array}\right)
$$

Thus, each multiplier $M$ is an upper triangular Toeplitz matrix and can be identified with a sequence $\left\{\alpha_{k}\right\}_{k=0}^{\infty}$ of complex numbers. The following theorem shows that each multiplier is in fact a bounded Toeplitz operator on $l^{2}$.

Theorem 2.1. The matrix $M$ is a bounded multiplier of $G^{\infty}$ if and only if there exists $\varphi \in H^{\infty}$ such that

$$
\alpha_{k}=\frac{1}{2 \pi i} \int_{0}^{2 \pi} \varphi\left(e^{i \theta}\right) e^{i k \theta} d \theta, \quad \text { for } \quad k=0,1,2, \ldots
$$

where $\left\{\alpha_{k}\right\}_{k=0}^{\infty}$ corresponds to $M$ as in (2.1). Moreover, $\|M\|=\|\varphi\|_{\infty}$. 
Proof. Let $M$ be a (bounded) multiplier and $\Gamma_{n}=\Gamma\left(z^{-n}\right)$. the matrix corresponding to $z^{-n}$ by Theorem 1.1. Then $M \Gamma_{n}=\Gamma\left(z^{-n} \varphi_{n}\right)$ where $\varphi_{n}(z)=\sum_{k=0}^{n-1} \alpha_{k} z^{k}$. Since

$$
\operatorname{dist}\left(z^{-n} \varphi_{n}, H^{\infty}\right)=\left\|M \Gamma_{n}\right\| \leqq\|M\|,
$$

it follows that there exists $h_{n} \in H^{\infty}$ such that $\left\|z^{-n} \varphi_{n}-h_{n}\right\|_{\infty} \leqq\|M\|$ for $n=1,2, \ldots$. Thus the sequence $\left\{\varphi_{n}-z^{n} h_{n}\right\}_{n=1}^{\infty}$ has a weak $*$ limit $\varphi$, where $\varphi \in H^{\infty}$. It is clear that $\varphi$ satisfies (2.2).

Now suppose $\varphi \in H^{\infty}$ where $\varphi(z) \sim \sum_{k=0}^{\infty} \alpha_{k} z^{k}$. Let $M_{\varphi}=M$, where $M$ corresponds to $\left\{\alpha_{k}\right\}_{k=0}^{\infty}$ as in (2.1). If $\Gamma=\Gamma_{f}=\left(\gamma_{j+k-1}\right) \in G^{\infty}$, then since $\varphi f \in L^{\infty}$, it follows that $\Gamma_{\varphi f}=\left(\gamma_{j+k-1}^{\prime}\right) \in G^{\infty}$ where

$$
\gamma_{k}^{\prime}=\sum_{j=0}^{\infty} \alpha_{j} \gamma_{k+j} .
$$

However, the matrix $\left\{\gamma_{k+j-1}^{\prime}\right\}$ coincides with formal product $M_{\varphi} \Gamma$. Thus $M_{\varphi}$ is a multiplier of $G^{\infty}$. Now as we have just shown $M_{\varphi} \Gamma_{f}=\Gamma_{\varphi f}$, and so since

$$
\left\|\Gamma_{\varphi f}\right\|=\operatorname{dist}\left(\varphi f, H^{\infty}\right) \leqq\|\varphi\|_{\infty} \operatorname{dist}\left(f, H^{\infty}\right)=\|\varphi\|_{\infty}\left\|\Gamma_{f}\right\|,
$$

we have $\left\|M_{\varphi}\right\| \leqq\|\varphi\|_{\infty}$ and hence $M_{\varphi}$ is bounded. The following lemma completes the proof of the theorem.

Lemma 2.2. Given $\varphi \in H^{\infty},\|\varphi\|_{\infty}=1$ and $\varepsilon>0$ there exists $F \in L^{\infty},\|F\|_{\infty}=1$ such that $\operatorname{dist}\left(\varphi F, H^{\infty}\right) \geqq 1-\varepsilon$.

Proof. Let $\varepsilon_{1}>0$ be given. Choose a sequence $\left\{z_{n}\right\}_{n=1}^{\infty}$ from the open unit disk $D$ such that $\lim _{n \rightarrow \infty} \varphi\left(z_{n}\right)=w_{0}$, where $1-\varepsilon_{1} \leqq\left|w_{0}\right| \leqq 1$ and where

1. $\left|w_{0}-\varphi\left(z_{n}\right)\right|<\varepsilon_{1}$ and,

2. $1-\left|z_{n+1}\right| \leqq 1 / 2\left(1-\left|z_{n}\right|\right)$ for $n=1,2, \ldots$.

The sequence $\left\{z_{n}\right\}_{n=1}^{\infty}$ is therefore uniformly separated and so [6, 9 p. 148] there exists $G \in H^{\infty},\|G\|_{\infty} \leqq k \varepsilon_{2}$ such that

$$
G\left(z_{n}\right)=\frac{w_{0}-\varphi\left(z_{n}\right)}{w_{0} \varphi\left(z_{n}\right)} \text { for } n=1,2, \ldots, \quad \text { where } \quad \varepsilon_{2}=\frac{\varepsilon_{1}}{\left(1-\varepsilon_{1}\right)\left(1-2 \varepsilon_{1}\right)}
$$

and $k$ is independent at $\varepsilon_{2}$. Now set $F(z)=\bar{B}(z) g(z)$ where

The function

$$
B(z)=\prod_{n=1}^{\infty} \frac{\left|z_{n}\right|}{z_{n}}\left(\frac{z_{n}-z}{1-\bar{z}_{n} z}\right), \quad \text { and } \quad g(z)=\frac{w_{0}^{-1}+G(z)}{\left\|w_{0}^{-1}+G\right\|_{\infty}} .
$$

$$
h(z)=\frac{\left\|w_{0}^{-1}+G\right\|_{\infty}^{-1}-g(z) \varphi(z)}{B(z)} \in H^{\infty}
$$

and is in fact the best $H^{\infty}$ approximation to $\varphi F$. This follows since

which implies that

$$
\varphi F-h=\left\|w_{0}^{-1}+G\right\|_{\infty}^{-1} \overline{B(z)}
$$

$$
\operatorname{dist}\left(\varphi F, H^{\infty}\right) \geqq 1-\frac{k \varepsilon_{2}}{1-\varepsilon_{1}} .
$$




\section{Properties of multipliers}

Each $\varphi \in H^{\infty}$ defines a bounded linear operator from $G^{\infty}$ into $G^{\infty}$ by $\Gamma \mapsto M_{\varphi} \Gamma$. The following proposition relates the properties of the operator $M_{\varphi}$ to properties of the corresponding $H^{\infty}$ function $\varphi$.

Proposition 3.1. Let $\varphi \in H^{\infty}$. Then

1. $M_{\varphi}$ is onto $G^{\infty}$ if and only if the outer part of $\varphi$ is invertible in $H^{\infty}$,

2. the inverse $\left(M_{\varphi}\right)^{-1}$ exists and is a (bounded) multiplier if and only if $\varphi$ is invertible in $H^{\infty}$,

3. $M_{\varphi}$ is one to one if and only if $\varphi$ is an outer function and,

4. if $\varphi$ has a non-constant inner factor $s$, then the kernel of $M_{\varphi}$ is the set of matrices corresponding to $\bar{s} H^{\infty}$.

Proof. 1. Let $\varphi \in H^{\infty}$, then we may write $\varphi=s k$, where $s$ is an inner function and $k$ is an outer function. Assume $k^{-1} \in H^{\infty}$. To show $M_{\varphi}$ is onto, it suffices to show by Theorem 1.1 that every $f \in L^{\infty}$ can be written as $\varphi g$ for some $g \in L^{\infty}$. The implication follows if we let $g=f \bar{s} k^{-1}$. Now suppose $M_{\varphi}$ is onto and $k^{-1} \notin H^{\infty}$. Then there exists a sequence $\left\{\alpha_{k}\right\}_{k=1}^{\infty} \in D$ such that $k\left(\alpha_{j}\right) \underset{j \rightarrow \infty}{\longrightarrow} 0$ and $\sum_{j=1}^{\infty}\left(1-\left|\alpha_{j}\right|\right)<\infty$. Let

$$
B(z)=\prod_{j=1}^{\infty} \frac{\left|\alpha_{j}\right|}{\alpha_{j}}\left(\frac{\alpha_{j}-z}{1-\bar{\alpha}_{j} z}\right)
$$

and suppose there exists $f \in L^{\infty}$ such that $M_{\varphi} \Gamma_{f}=\Gamma_{\bar{B}}$. This would imply that $\varphi f+h=\bar{B}$ for some $h \in H^{\infty}$, and hence $k g=1-B h \in H^{\infty}$ where $g=s B f$. Write $k g=s_{1} k_{1}$, where $s_{1}$ is inner and $k_{1}$ is outer. Set $k_{2}=k_{1} / k$. Then $g=s_{1} k_{2}$ and so $g \in H^{\infty}$. However this is impossible since $k g\left(\alpha_{j}\right) \rightarrow 1$ and $k\left(\alpha_{j}\right) \rightarrow 0$.

2. Suppose $M_{\varphi}$ is a (bounded) multiplier with an inverse $\left(M_{\varphi}\right)^{-1}$ which is also a (bounded) multiplier. Then $\left(M_{\varphi}\right)^{-1}=M_{\psi}$ for some $\psi \in H^{\infty}$. Since $(\Gamma \xi, \eta)=$ $\left(M_{\varphi} M_{\psi} \Gamma \xi, \eta\right)$ for all $\Gamma \in G^{\infty}$ and $\xi, \eta \in l^{2}$ we have [1] that

$$
\int_{0}^{2 \pi} \psi \varphi F g d \theta=\int_{0}^{2 \pi} F g d \theta \text { for all } F \in L^{\infty} \text { and } g \in H_{\mathbf{0}}^{1} .
$$

Hence $\psi \varphi=1$ a.e. which proves our assertion.

Conversely, if $\varphi^{-1} \in H^{\infty}$, then $M_{\varphi-1}$ is a (bounded) multiplier, and by the previous argument $\left(M_{\varphi-1} M_{\varphi} \Gamma \xi, \eta\right)=(\Gamma \xi, \eta)$ for all $\xi, \eta \in l^{2}$ and for all $\Gamma \in G^{\infty}$. Therefore $\left(M_{\varphi}\right)^{-1}=M_{\varphi-1}$.

3 and 4. Again let $\varphi=s k$ where $s$ is inner, $k$ is outer and suppose there exists $f_{1}, f_{2} \in L^{\infty}$ such that: $M_{\varphi} \Gamma_{f_{1}}=M_{\varphi} \Gamma_{f_{2}}$. This implies $s\left(f_{1}-f_{2}\right)=h k^{-1}$ where $h \in H^{\infty}$. A similar argument as that in the proof of part (1) shows that $s\left(f_{1}-f_{2}\right) \in H^{\infty}$ which implies that $\left(f_{1}-f_{2}\right) \in \bar{s} H^{\infty}$. Thus we see that if $\varphi$ is outer, i.e. $s$ is constant, then $f_{1}-f_{2} \in H^{\infty}$ and so $\Gamma_{f_{1}}=\Gamma_{f_{2}}$. 


\section{Diminishing multipliers}

Theorem 4.1. Given any $\Gamma_{F} \in G^{\infty}$ and $\varepsilon>0$ there exists a multiplier $M_{\varphi}$ such that $\left\|M_{\varphi} \Gamma_{F}\right\|<\varepsilon$.

Proof. We may assume without any loss of generality that $\left\|\Gamma_{F}\right\|<1$ and [2] that $|F|=1$ a.e. There exists Blaschke products $B_{1}, B_{2}$ such that $\left\|F-B_{1} \bar{B}_{2}\right\|_{\infty}<\varepsilon$ [8] and so if we let $\varphi=B_{2}$ it then follows that $\left\|M_{\varphi} \Gamma_{F}\right\|=\left\|\Gamma_{\varphi F}\right\|<\varepsilon$.

The proof of the last theorem shows that every $\Gamma \in G^{\infty}$ can be ,diminished" by multipliers of a special type, namely, $M_{\varphi}$ where $\varphi$ is an infinite Blaschke product. This naturally leads to the question of whether a given matrix $\Gamma$ can be arbitrarily reduced in norm by multiplication with even more elementary multipliers. In particular, multipliers of the form $M_{\varphi}$ where $\varphi$ is a finite Blaschke product. Such a multiplier corresponds to a matrix which is the finite product of matrices of the form:

$$
K_{\alpha}\left(\begin{array}{ccccc}
\frac{\alpha^{2}}{|\alpha|^{2}-1} & \alpha & \alpha^{2} & \alpha^{3} & \ldots \\
0 & \frac{\alpha^{2}}{|\alpha|^{2}-1} & \alpha & \alpha^{2} & \ldots \\
0 & 0 & \frac{\alpha^{2}}{|\alpha|^{2}-1} & \alpha & \ldots \\
0 & 0 & 0 & \frac{\alpha^{2}}{|\alpha|^{2}-1} & \ldots \\
\vdots & \vdots & \vdots & \vdots & \ddots
\end{array}\right)
$$

where

$$
K_{\alpha}=-\bar{\alpha}\left(\frac{|\alpha|^{2}-1}{|\alpha|^{2}}\right) \text { and } 0<|\alpha|<1 \text {. }
$$

If this product contains $n$ terms, that is if $\varphi$ is a finite Blaschke product of degree $n$, then we call $M_{\varphi}$ a geometric multiplier of degree $n$. Let the set of all geometric multipliers of degree $n$ be denoted by $\mathscr{M}_{n}$.

It is obvious that given any $\Gamma \in G^{\infty}$ and any $M \in \mathscr{M}_{n}$ then $\|M \Gamma\| \leqq\|\Gamma\|$ and hence every multiplier in $\mathscr{M}_{n}$ can be viewed as a diminishing multiplier for $G^{\infty}$.

The next theorem establishes a relationship between diminishing geometric multipliers and approximation by generalized rational functions.

Theorem 4.2. For each $\Gamma_{F} \in G^{\infty}$,

$$
\inf _{M_{\varphi} \in \mathscr{M}_{n}}\left\|M_{\varphi} \Gamma_{F}\right\|=\inf _{\substack{h \in H \\ p_{n} \in \mathscr{P}_{n}}}\left\|F-\frac{h}{p_{n}}\right\|_{\infty}
$$

where $\mathscr{P}_{n}$ denotes the space of algebraic polynomials of degree $n$ or less. 
Proof. If $M_{\varphi} \in \mathscr{M}_{n}$ then $\varphi$ is a finite Blaschke product of degree $n$ and so

$$
\begin{aligned}
\inf _{M_{\varphi} \in \mathscr{M}_{n}}\left\|M_{\varphi} \Gamma_{F}\right\| & =\inf _{\varphi \in \mathscr{B}_{n}} \operatorname{dist}\left(\varphi F, H^{\infty}\right) \\
& =\inf _{\varphi \in \mathscr{B}_{n}} \operatorname{dist}\left(F, \bar{\varphi} H^{\infty}\right) \\
& =\inf _{\substack{h \in H^{\infty} \\
\left|a_{k}\right|<1 \\
k=1,2, \ldots, n}}\left\|F-\frac{h}{\prod_{k=1}^{n}\left(z-a_{k}\right)}\right\|_{\infty}
\end{aligned}
$$

where $\mathscr{B}_{n}$ denotes the set of finite Blaschke products of degree $n$. Now in each case $F \in L^{\infty}$ we have that

$$
\inf _{\substack{\left|a_{k}\right|<1 \\ k=1, \ldots, n}}\left\|F-\frac{h}{\prod_{k=1}^{n}\left(z-a_{k}\right)}\right\|_{\infty}=\inf _{p_{n} \in \mathscr{P}_{n}}\left\|F-\frac{h}{p_{n}}\right\|_{\infty} .
$$

If we apply a result obtained by Adamjan, Arov and Kreĭn in their paper [3] in which they extensively studied the problem of approximating a given $F \in L^{\infty}$ by functions of the form $h / p_{n}, h \in H^{\infty}, p_{n} \in \mathscr{P}_{n}$, and its relationship to the properties of $\Gamma_{F}$ as an operator, we can state the following

Corollary 4.3. Given any $\Gamma \in G^{\infty}$ and $n \in N$, there exists $M^{0} \in \mathscr{M}_{n}$ for which $\inf _{M \in \mathscr{M}_{n}}\|M \Gamma\|$ is attained. Moreover, $\left\|M^{0} \Gamma\right\|=S_{n}(\Gamma)$, where $S_{n}(\Gamma)$ is the $n$-th $s$ number of $\Gamma[3]$.

We are naturally led to ask for which Hankel matrices $\Gamma$, $\lim _{n \rightarrow \infty} \inf _{\varphi \in \mathscr{B}_{n}}\left\|M_{\varphi} \Gamma\right\|=0$, since as we have already observed, given any $\Gamma$, $\inf \left\|M_{\varphi} \Gamma\right\|=0$ where the inf is taken over all Blaschke products.

Theorem 4.4. If $\Gamma_{F} \in G^{\infty}$, then $\lim _{n \rightarrow \infty} \inf _{M \in M_{n}}\left\|M \Gamma_{F}\right\|=0$ if and only if $F \in H^{\infty}+C$, where $C$ is the algebra of continuous functions on $\partial D$.

Proof. If $\lim _{n \rightarrow \infty} \inf _{M \in \mathscr{M}_{n}}\left\|M \Gamma_{F}\right\|=0$, then there exists a sequence of functions $\left\{h_{k}+r_{k}\right\}_{k=1}^{\infty}$ where $h_{k} \in H^{\infty}, r_{k}$ is a rational function having all of its poles in $D$, and such that $\lim _{k \rightarrow \infty}\left\|F-\left(h_{k}+r_{k}\right)\right\|_{\infty}=0$.

The functions $h_{k}+r_{k} \in H^{\infty}+C$ and hence [11] since $H^{\infty}+C$ is closed the sufficient portion of our theorem follows.

The converse follows immediately upon applying Weierstrass's theorem. 


\section{Remarks}

As we mentioned in Section 2, it is not necessary to assume that a multiplier $M_{\varphi}$ is bounded. That is, if $M_{\varphi}$ is of the form (2.1) and $M_{\varphi} \Gamma \in G^{\infty}$ for all $\Gamma \in G^{\infty}$, then

$$
\sup _{\substack{\Gamma \in G^{\infty} \\\|\Gamma\|<1}}\left\|M_{\varphi} \Gamma\right\|<\infty .
$$

This is not difficult to demonstrate for such a multiplier must by necessity also be an operator defined on all of $l^{2}$ and so is a bounded operator since it has a matrix representation $[4$, p. 50$]$. (The same reasoning shows that any "Hankel" operator is bounded.) Let

$$
\left\|M_{\varphi}\right\|_{\text {op }}=\sup _{\substack{\xi \in l^{2} \\\|\xi\|_{2} \leqq 1}}\left\|M_{\varphi} \xi\right\|
$$

Then

$$
\left\|M_{\varphi}\right\| \equiv \sup _{\substack{\Gamma \in G^{\infty} \\\|\Gamma\| \leqq 1}}\left\|M_{\varphi} \Gamma\right\|=\sup _{\substack{\Gamma \in G^{\infty} \\\|\Gamma\| \leqq 1}} \sup _{\substack{\xi \in l^{2} \\\|\|_{2} \leqq 1}}\left\|M_{\varphi} \Gamma \xi\right\| \leqq\|M \varphi\|_{\mathrm{op}}
$$

One can in fact show that $\left\|M_{\varphi}\right\|=\left\|M_{\varphi}\right\|_{\text {op }}$ for if $M_{\varphi}$ is a (bounded) multiplier then $\varphi \in H^{\infty}$ and so the Toeplitz operator $M_{\varphi}$ has norm $\left\|M_{\varphi}\right\|_{\text {op }}=\|\varphi\|_{\infty}[$ [7, p. 179]. Our claim now follows since by Theorem 2.1, $\left\|M_{\varphi}\right\|=\|\varphi\|_{\infty}$.

A problem which arose in proving Theorem 2.1 which seems to be open is whether given any $F \in L^{\infty}$ with $\|F\|_{\infty}=1$, does there exist $G \in L^{\infty}$ such that $|G|=|F|$ a.e. and $\operatorname{dist}\left(G, H^{\infty}\right)=1$.

It is not difficult to prove this in the case where $|F|=1$ on some arc. One can also view Lemma 2.2 as a partial solution to this problem.

\section{References}

[1] Adamyan, V. M., D. Z. Arov, and M. G. Krě̆n: Infinite Hankel Matrices and generalized problems of Carathéodory-Fejér and F. Riesz - Funkcional. Anal. i Priložen. 2:1, 1968, 1-19 (Russian).

[2] Adamjan, V. M., D. Z. Arov, and M. G. Kreľn: Infinite Hankel matrices and generalized problems of Carathéodory-Fejér and I. Schur. - Funkcional. Anal. i Priložen. 2:4, 1968, 269-281 (Russian).

[3] Adamjan, V. M., D. Z. Arov, and M. G. KreǏn: Analytic properties of the Schmidt pairs of a Hankel operator and the generalized Schur-Takagi problem. - Math. USSR-Sb. $15: 1,1971,31-72$.

[4] Akhiezer, N. I., and I. M. Glazman: Theory of linear operators in Hilbert space. Vol. I. Frederick Ungar Publishing Co., N. Y., 1961.

[5] Brown, A., and P. R. Halmos: Algebraic properties of Toeplitz operators. - J. Reine Angew. Math. 213, 1971, 89-102.

[6] Carleson, L.: An interpolation problem for bounded analytic functions. - Amer. J. Math. $80,1958,921-930$. 
[7] Douglas, R. G.: Banach algebra techniques in operator theory. - Academic Press, N. Y., 1972.

[8] Douglas, R. G., and W. Rudin: Approximation by inner functions. - Pacific J. Math. 31, 1969, 313-320.

[9] Duren, P. L.: Theory of $H^{p}$ spaces. - Academic Press, N. Y., 1970.

[10] NeHARI, Z.: On bounded bilinear forms. - Ann. of Math. (2) 65, 1957, 153-162.

[11] Sarason, D.: Algebras of functions on the unit circle. - Bull. Amer. Math. Soc. 79, 1973, 286-299.

\section{Clark University}

Department of Mathematics

Worcester, Massachusetts 01610

USA

Received 26 November 1979 